\title{
Molecularly Imprinted Sol-Gel Nanotubes Membrane for Biochemical Separations
}

\author{
Huang-Hao Yang ${ }^{*}$, , Shu-Qiong Zhang ${ }^{\ddagger}$, Wei Yang ${ }^{\ddagger}$, Xiao-Lan Chen ${ }^{\ddagger}$, Zhi-Xia Zhuang ${ }^{\dagger, \star}$, \\ Jin-Gou Xu ${ }^{\ddagger}$, Xiao-Ru Wang ${ }^{\dagger, \ddagger}$ \\ Material Transport \& Transformation in Environmental \& Life Processes Lab, The First \\ Institute of Oceanography, SOA, Qingdao, 266061, P.R. China and The Key Laboratory \\ of Analytical Sciences of MOE, Department of Chemistry, Xiamen University, Xiamen \\ 361005, P.R.China
}

\section{Supporting Information}

Materials. 3-(Triethoxysilyl)propyl isocyanate, tetraethoxysilane (TEOS), dibutyltin dilaurate (DBDU) and 3-aminopropyltriethoxysilane were purchased form Aldrich Chemical Company. Estrone and testosterone propionate were purchased from Sigma. THF, chloroform and DMSO were used after purification by standard method. Other chemicals were used as received without further purification.

Synthesis of silica monomer-estrone complex. Estrone $(1 \mathrm{~g}, 3.7 \mathrm{mmol})$ was dissolved in THF $(30 \mathrm{~mL})$. To this solution, 3-(triethoxysilyl)propyl isocyanate $(2 \mathrm{~mL}, 8.1 \mathrm{mmol})$ and DBDU $(2 \mathrm{~mL})$ were added at room temperature. The reaction mixture was stirred for $24 \mathrm{~h}$ at $75{ }^{\circ} \mathrm{C}$. The solvent was evaporated and the product was isolated by column chromatography on silica gel (ethyl acetate : hexane $=1: 3$ ). Yield: $65 \%$.

Preparation of estrone-silica nanotubes membrane. TEOS $(0.28 \mathrm{~g}, 1.35 \mathrm{mmol})$ and silica monomer-estrone complex $(14 \mathrm{mg}, 67.6 \mu \mathrm{mol})$ were added to a mix solution of 1 $\mathrm{mL}$ ethanol and $19 \mathrm{~mL}$ acetate buffer $(0.1 \mathrm{~mol} / \mathrm{L}, \mathrm{pH}=5.1)$. The resulting solution was stirred for $5 \mathrm{~min}$, and the alumina membrane was then immersed. After $0.5 \mathrm{~h}$ in low pressure, the membrane was removed from the solution, rinsed with ethanol, and cured at $150{ }^{\circ} \mathrm{C}$ in air for $1 \mathrm{~h}$.

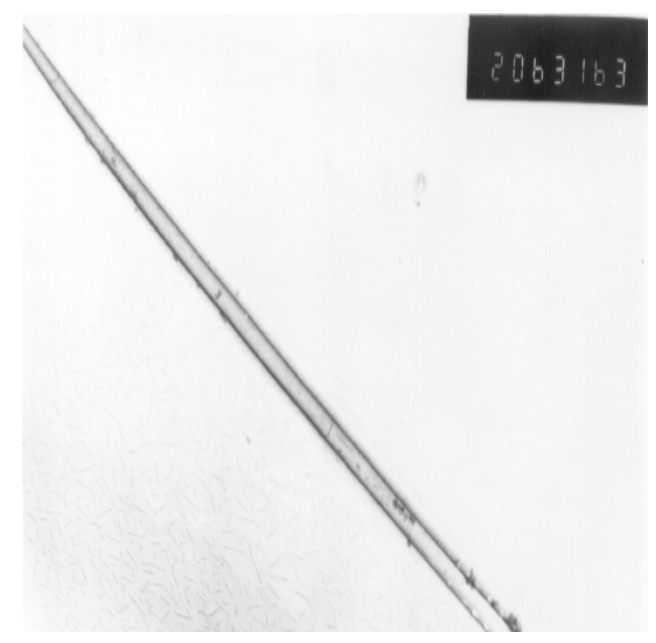

Figure S1. Another TEM micrograph of $\mathrm{SiO}_{2}$ nanotubes prepared in this experiment 
Preparation of molecularly imprinted silica nanotubes membrane. The estrone-silica nanotubes membrane was added to a mix solution of DMSO $(15 \mathrm{~mL})$ and water $(3 \mathrm{~mL})$. The mixture was heated at $180{ }^{\circ} \mathrm{C}$ under low pressure for $5 \mathrm{~h}$. Then the membrane was placed into a springe filter holder. DMSO and water mixture solution was further passed through this device to fully elute the residual estrone for 1 day.

Preparation of a Control Silica Nanotubes Membrane. Control silica nanotubes membrane was synthesized in the almost same manner for the preparation of molecularly imprinted silica nanotubes membrane except that phenol replaced estrone. The procedure includes synthesis of silica monomer-phenol complex, preparation of phenol-silica nanotubes membrane and thermolysis.

\section{Molecule Structure of Testosterone Propionate.}

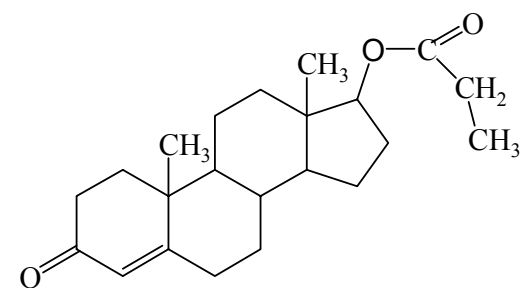

Steady-state Binding Experiment. Six pieces of molecularly imprinted silica nanotubes membranes and six pieces of control silica nanotubes membranes were synchronously prepared under the same experimental. The molecularly imprinted nanotubes membranes and control nanotubes membranes were placed into a springe filter holder described above, respectively. These nanotubes membranes were mounted into a circulative flow system, and the test solutions of estrone or testosterone propionate (at various concentrations) in $20 \mathrm{~mL}$ chloroform were passed through the nanotubes membranes by a peristaltic pump for $12 \mathrm{~h}$, respectively. The filtrates were concentrated by the evaporation. The amounts of estrone adsorbed by the molecularly imprinted silica nanotubes membranes were determined by measuring the residual estrone in filtrates by competitive indirect enzyme-linked immunoassay.

Measuring the Affinity of Estrone to Molecularly Imprinted Silica Nanotubes versus Control Silica Nanotubes. Three pieces of molecularly imprinted silica nanotubes membranes and three pieces of control silica nanotubes membranes were synchronously prepared under the same experimental conditions. The molecularly imprinted nanotubes membranes and control nanotubes membranes were placed into the assembly devices describe above, respectively. These nanotubes membrane assembles were mounted into a circulative flow system, and the test solutions of estrone in $20 \mathrm{~mL}$ chloroform at concentration of $3 \mathrm{mM}$ were passed through the nanotubes membrane by a peristaltic pump for $12 \mathrm{~h}$, respectively. The amounts of estrone adsorbed by molecularly imprinted silica nanotubes membranes were determined by measuring the residual estrone in filtrates by competitive indirect enzyme-linked immunoassay. The value of affinity is an average value of three times parallel determinations. 
Kinetic Binding Profile. A piece of molecularly imprinted silica nanotubes membrane was placed into the assembly device described above. This nanotubes membrane assemble was placed into a circulative flow system, and the test solution of estrone in 20 $\mathrm{mL}$ chloroform at concentration of $3 \mathrm{mM}$ was passed through the nanotubes membrane by a peristaltic pump for $12 \mathrm{~h}$. In this period, a series of $50 \mu \mathrm{L}$ of the efflux solutions were intermittently taken out and detected by competitive indirect enzyme-linked immunoassay. The results were shown in Figure S2.

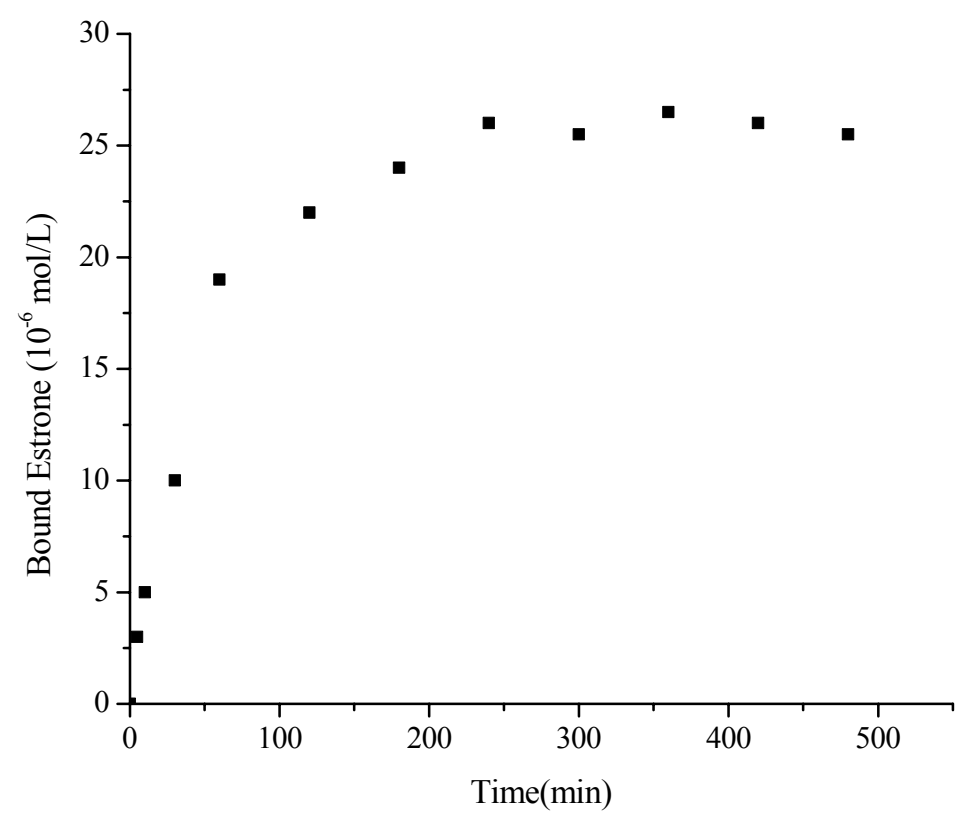

Figure S2. Kinetic binding profile of estrone binding to the molecularly imprinted nanotubes membrane 